\title{
$\Delta$ 9-tetrahydrocannabinol (THC) vapor exposure produces conditioned rewarding effects in male and female rats
}

\author{
Catherine F. Moore ${ }^{a^{*}}$, Catherine M. Davis ${ }^{b}$, Elise M. Weerts ${ }^{a}$
}

a. Division of Behavioral Biology, Department of Psychiatry and Behavioral Sciences, Johns Hopkins University School of Medicine

b. Department of Pharmacology and Molecular Therapeutics, Uniformed Services University of the Health Sciences, Bethesda, MD.

* Corresponding author at: Johns Hopkins Bayview Research Campus, Behavioral Biology

Research Center, 5510 Nathan Shock Drive, Suite 3000, Baltimore, MD 21224, USA. E-mail address: cfmoore@jhmi.edu (C.F. Moore). 


\section{Abstract}

Background: Vaping of cannabis and cannabis extracts containing $\Delta 9$-tetrahydrocannabinol (THC, the primary psychoactive constituent of cannabis) is on the rise. Development of animal models using vapor exposure is important for increasing our understanding of the rewarding and aversive effects of vaped cannabinoids. Currently there are limited data on the conditioned rewarding effects of THC vapor in rats, and no studies to date examining sex differences. Methods: Male and female Sprague-Dawley rats (N=96; 12 per sex/group) underwent place conditioning after exposure to vaporized THC or vehicle (propylene glycol, PG). THC vaporconditioned rats received one of three THC vapor exposure amounts (THC Group 1:5 puffs of $100 \mathrm{mg} / \mathrm{ml} \mathrm{THC}$, THC Group 2: 5 puffs of $200 \mathrm{mg} / \mathrm{ml} \mathrm{THC,} \mathrm{or} \mathrm{THC} \mathrm{Group} \mathrm{3:} 10$ puffs of 200 $\mathrm{mg} / \mathrm{ml} \mathrm{THC}$ ) and matched vehicle vapor (PG) exposure and on alternate days for two sets of 8 daily sessions (16 days total). Vehicle-conditioned rats (Veh Group 0) received only PG vapor exposure each day. Rats were passively administered vapor for 30-min immediately before daily, 30-min conditioning sessions. Untreated rats completed place preference tests after the 8th and 16th conditioning sessions and then testing continued daily until extinction occurred. Following extinction, rats underwent a THC vapor-primed reinstatement session.

Results: Male and female rats showed an exposure-dependent preference for the THC vaporpaired chamber, though sex differences were observed. The lowest THC vapor exposure group tested (THC Group 1) did not produce CPP in males or females. Exposure to the middle condition tested (THC Group 2) resulted in preference for the THC vapor-paired chamber in males, but not females. The highest THC vapor exposure condition tested (THC Group 3) produced place preference in both males and females. Preference for the THC-paired chamber extinguished more quickly in males than in females. Following extinction, THC vapor reexposure (i.e., drug-prime) did not result in reinstatement of place preference for either sex.

Conclusion: This study demonstrated conditioned rewarding effects of THC vapor in both male and female rats, and provides evidence for sex differences in doses of THC vapor that produce 
CPP and in time to extinction. Conditioned place aversion was not observed at any of the THC vapor exposure amounts tested. These data provide a foundation for future exploration of the conditioned rewarding effects vaporized THC, cannabis and its constituents in preclinical models.

Keywords: THC; Vapor Exposure; Conditioned Place Preference; Reward; Cannabis Abbreviations: $\Delta 9$-tetrahydrocannabinol (THC); Conditioned Place Preference (CPP); Conditioned Place Aversion (CPA); Propylene Glycol (PG) 


\section{Introduction}

Vaping of cannabis and cannabinoids such as $\Delta 9$-tetrahydrocannabinol (THC, the primary psychoactive constituent of cannabis) is on the rise (Budney et al., 2015; Giroud et al., 2015; Morean et al., 2015; Ramo et al., 2015; Varlet et al., 2016). Recent estimated rates of vaping cannabis are reported to be between $20 \%-37 \%$ (past 30-days) and 60\% (lifetime) in cannabis users in the US (Goodman et al., 2020; Lee et al., 2016; Schauer et al., 2020). Vaping has also been promoted as a safer alternative to smoked cannabis (Budney et al., 2015). At the same time, a wide array of new products of cannabis constituents for vaping are now available, with little data available on their addictive potential. The use of rat vapor models to examine the rewarding effects of vaporized cannabis constituents can increase our understanding of their potential abuse liability.

Place conditioning procedures can reveal the conditioned rewarding or aversive effects of an administered substance. In this procedure, drug is administered followed with pairing in a distinct environment with various contextual clues. On alternate conditioning sessions, vehicle administration (no drug) is paired with a separate, distinct environment. On a drug- and vehiclefree test day, animals are allowed unrestricted access to both environments to assess conditioned place preference (CPP) or aversion (CPA). Increases in time spent on the drugpaired side (i.e., preference) indicates conditioned rewarding effects of the drug, and decreases in time spent on the drug-paired side indicates conditioned aversive effects of the drug. Following the acquisition of CPP, repeated, drug-free extinction tests can be conducted to assess the persistence of preference to the conditioned chamber (Aguilar et al., 2009). To assess relapse-like behavior, reinstatement test can be conducted following exposure to a stress-, cue-, or drug- induced priming (Shaham et al., 2003).

To date, the majority of research examining the conditioned rewarding effects of cannabis and cannabinoids have primarily used intraperitoneal, intravenous, or subcutaneous routes of administration (Murray and Bevins, 2010), with vapor exposure models only more 
recently being developed and used (Moore et al., 2020). Depending on route of administration, THC can produce markedly different physiological and behavioral effects, due to distinctive pharmacokinetics and metabolic pathways (e.g., second pass metabolism)(Hlozek et al., 2017; Spindle et al., 2020). In tests of conditioned effects of THC when injected, low doses (0.075-4 $\mathrm{mg} / \mathrm{kg}$ ) can produce preference, while higher doses $(5-20 \mathrm{mg} / \mathrm{kg}$ ) often produce aversion (for reviews, see Kubilius et al., 2018; Panagis et al., 2008).

Previously we evaluated the antinociceptive, hypothermic, and appetitive effects of a range of THC vapor exposure conditions in two strains of males and female rats (Moore et al., 2021). We demonstrated effects were orderly, THC vapor exposure dependent, and also identified sex differences. Lower THC vapor exposure amounts increased appetitive effects in a test of progressive ratio responding for food pellet rewards, while high THC vapor exposure amounts resulted in hypothermia, antinociception, and sedative effects (Moore et al., 2021). The current study sought to evaluate whether the THC vapor exposure conditions equivalent to those that produced appetitive effects in our prior study would produce CPP or CPA. The current study design included both male and female rats and an 'exposure-effect' curve with multiple THC exposure groups to assess CPP. Second, we evaluated extinction and drugprimed reinstatement of CPP.

\section{Materials and Methods}

\subsection{Subjects}

Adult male and female Sprague Dawley rats ( $N=96,12$ per sex/group)(Charles River, Wilmington, MA), 8 weeks old at the start of experiments, were single housed in wire-topped, plastic cages $(27 \times 48 \times 20 \mathrm{~cm})$ with standard enrichment. The vivarium was on a $12 \mathrm{hr}$ reverse light cycle (lights off at 8:00 a.m.) and was humidity and temperature controlled. Diet was a corn-based chow (Teklad Diet 2018; Harlan, Indianapolis, IN); rats had ad libitum access to food and water except during test procedures. All procedures used in this study were approved by 
the Johns Hopkins Institutional Animal Care and Use Committee. The facilities adhered to the National Institutes of Health Guide for the Care and Use of Laboratory Animals and were AAALAC-approved.

\subsection{Drugs}

THC stock solutions (100 and $200 \mathrm{mg} / \mathrm{ml}$ in 95\% ethanol) were provided by the U.S. National Institute on Drug Abuse Drug Supply Program. Ethanol from the THC stock was evaporated using nitrogen and then THC was mixed in $100 \%$ propylene glycol to yield a 100 or $200 \mathrm{mg} / \mathrm{ml}$ THC solution for vaporization.

\subsection{Vapor exposure system}

A commercial vapor chamber system (La Jolla Alcohol Research Institute, La Jolla CA) was used which contained four sealed polycarbonate rat cages ( $35 \times 28 \times 26 \mathrm{~cm}$; 25L) adapted for the delivery of vaporized drug via an electronic vapor device (Smok Baby Beast Brother TFV8 Sub-Ohm Tank with the V8 X-Baby M2 0.25- $\Omega$ coil; SMOKTech, Shenzhen, China) and connected to an air pump to regulate airflow. The chamber air was vacuum controlled by a pump which pulls room ambient air into the chamber through an intake valve and out through the exhaust valve at a constant rate $(2-3 \mathrm{~L} / \mathrm{min})$. The e-vape controller was set to a maximum temperature of $400^{\circ} \mathrm{F}(\sim 30 \mathrm{~W})$, which is the same temperature used for vaporization in our clinical research (Spindle et al., 2020).

\subsection{Conditioned Place Preference}

The CPP apparatus used contained 3-chambers each with distinct environments and automated recording of activity via infrared beam breaks (San Diego Instruments, Inc.). The left chamber had white walls and a textured plastic floor, the middle chamber has clear walls and a stainless steel, grid rod floor, and the right chamber had black walls and a smooth plastic floor. A biased 
CPP protocol was used. Prior to drug conditioning, rats were allowed to freely explore the 3chamber apparatus for $15 \mathrm{~min}$. To test the rewarding effects of THC vapor exposure, rats were then allocated into four groups in which rats received either $100 \%$ propylene glycol (vehicle condition), or one of three different THC vapor exposure conditions THC as detailed in Table 1. Rats were passively exposed to either the assigned THC vapor exposure amount or a matched vehicle vapor condition on alternating days (THC, VEH, THC etc.; see Fig 1) and confined to one chamber for $30 \mathrm{~min}$ (THC vapor = unbiased chamber; vehicle vapor $=$ biased chamber). Vehicle-conditioned rats received vehicle vapor exposure each day. Rats underwent two sets of 8 daily conditioning sessions (16 days total). Tests for CPP occurred after the 8th and 16th conditioning sessions and were identical to the pre-test. Criteria for place preference, determined a priori, was an overall change in preference for the THC vapor-paired chamber that is greater than 0 (i.e. no change). In the event of a place preference after the $16^{\text {th }}$ conditioning session, daily preference tests continued for that group until extinction criteria were met (change in preference $=0$ ). Vehicle vapor control groups were run for an equal number of extinction days as THC groups for comparison. Following extinction, rats underwent a drug-primed reinstatement session; each rat was exposed to the same vapor exposure parameters they received during the conditioning phases prior to a 15-min preference test (see Fig 1 for a timeline of test procedures).

\subsection{Data Analysis}

A CPP score was calculated (time in THC vapor paired chamber - time in unpaired chamber). Criteria for determination of a place preference and extinction were defined a priori. A significant place preference for each group was defined as a change in preference score (CPP Score Pre-Test - CPP Score Post-Test) > 0, determined by one-sample t-test. Extinction criteria was two consecutive days of no preference (change in preference $=0$, determined by onesample t-test). To evaluate effects of group and sex (between subjects effects), two-way 
ANOVAs were conducted to analyze the percent of time spent on THC vapor-paired side (or the initially biased side in the case of Vehicle vapor controls). Dunnett's post hoc tests were used to analyze differences in outcomes between THC and vehicle groups. Comparisons between sex were determined with Sidak's post hoc tests. Statistics were performed in Statistica 11 and GraphPad Prism with $p \leq 0.05$ for significance.

\section{Results}

\subsection{Development of a preference for THC vapor-paired chamber}

After each post-test, preference was determined for each group using the a priori criteria described above. In males tested after 8 days of conditioning (i.e. four THC and four vehicle vapor exposures), no exposure condition produced a significant change in CPP score (i.e. place preference; p's $>0.05)$; however after 16 days of conditioning, we observed a place preference for the two highest exposure amounts (THC Group 2: $t(11)=2.940, p=0.0135$; THC Group 3: $t(11)=4.549, p=0.0008)$ (Fig 2). In females, 8 days of conditioning with the highest exposure amount (THC Group 3) was sufficient to produce place preference for the THC vapor-paired chamber $(t(11)=2.959, p=0.0130)$. After continued conditioning, CPP was still evident in this group at Post-Test $2(\mathrm{t}(11)=2.821, \mathrm{p}=0.0166)$, and no other female groups receiving THC vapor exposure demonstrated CPP (Fig 2).

\subsection{Extinction and reinstatement of preference for THC vapor-paired chambers}

After Post-Test 2, all groups that demonstrated CPP continued testing under extinction conditions (drug free) until there were two consecutive days where the group mean preference $=0$. Males in THC Group 2 showed a transient increase in preference on day 1 of extinction $(t(11)=3.942, p=0.0023)$ that then extinguished with additional sessions ( $p$ 's $>0.05$ on extinction day 2 and 3). Males in THC Group 3 showed immediate extinction of CPP ( $p$ 's $>0.05$ s on extinction day 1 and 2). Females in THC Group 3 continued to show preference for the THC 
vapor-paired chamber until the $3^{\text {rd }}$ and $4^{\text {th }}$ extinction sessions (Extinction day $1: t(11)=3.356$, $\mathrm{p}=0.0064$; Extinction day $2: \mathrm{t}(11)=3.106, \mathrm{p}=0.010)($ Fig 2$)$. However, upon closer analysis of the data following the end of the experimental protocol, one female in THC Group 3 was identified as a consistent outlier (>2 standard deviations below group mean on Post-Test 2 and Extinction days 1-4). If this female is removed, the mean change in CPP score for the remaining females on the last day tested is $152.7 \pm 54.09(\mathrm{t}(10)=2.824, \mathrm{p}=0.018)$, which suggests that resistance to extinction of CPP in females was even more prolonged. A drug-primed reinstatement did not result in reinstatement to the THC vapor-paired compartment in any group ( $p$ 's $>0.05$; Fig 2).

\subsection{Effects of Sex and THC Exposure Condition on Place Preference for THC Vapor}

ANOVA evaluation of the percent of time spent on the THC vapor-paired side during Post-Test 1 did not show a significant main effect of Group $(F(3,88)=2.439, p=0.0698)$, Sex ( $F$ $(1,88)=0.05, p=0.82)$, nor an interaction of $\operatorname{Group} \times \operatorname{Sex}(F(3,88)=2.106, p=0.1052)$ after 8 days of conditioning. Following an additional 8 days of conditioning, analysis of the percent time spent in the THC vapor-paired compartment during Post-Test 2 showed a main effect of Group $(F(3,88)=5.879, p=0.0010)$ and an interaction of $\operatorname{Sex} x \operatorname{Group}(F(3,88)=3.406$, $\mathrm{p}=0.0211$ )(Fig 3). Post hoc tests show that percent time spent on the THC vapor-paired side was higher than the vehicle controls for males in THC Groups 2 and 3 and females in THC Group 3 (p's $\leq 0.05$ ). Further, the percent time on the THC vapor-paired side was higher in males in THC Group 2 compared with females exposed to the same THC vapor amount $(p=0.02)$.

\subsection{Locomotor effects of acute and repeated THC vapor exposure}

To assess whether locomotor activity was altered after acute and intermittent THC exposure vs. vehicle vapor exposure groups, we analyzed distance traveled during conditioning sessions on day 1 , day 7 , and day 15 (THC exposure days for the THC groups). In an analysis 
of activity across the conditioning days $(1,7,15)$, with Sex and Group as between subjects factors, we observed an interaction of Day $x \operatorname{Sex} x \operatorname{Group}(F(6,176)=3.284, p=0.0043)$. When analyzed separately by sex, in males only there was a main effect of Day $(F(2,88)=10.56$, $\mathrm{p}<0.0001)$, and a trend for a Day $x$ Group interaction $(F(6,88)=1.982, p=0.0767)$, but no main effect of Group $(F(3,88)=0.741, p=0.5331)$. In males, post-hoc tests show that the activity of the vehicle vapor control group was higher on day 1 compared with day 7 and 15, suggesting a habituation to the CPP compartments over time in vehicle exposed controls, but not the THC groups. Post-hoc comparisons with activity on vehicle vapor exposure days (grand mean) showed no differences between groups (Fig 4). In females, there was a main effect of Day $(F(2$, $88)=31.62, p<0.0001)$ and $\operatorname{Group}(F(3,88)=6.729, p=0.0008)$, but no Day $x$ Group interaction $(F(6,88)=1.863, p=0.10)$. Activity was highest on day 1 , particularly in rats exposed to $\mathrm{THC}$ vapor (groups 2, 3 vs. 0), and this activity decreased over successive days (groups 0, 2, 3), though THC exposed females remained sensitive to the hyperlocomotive effect of $\mathrm{THC}$ exposure. Post-hoc comparisons with activity on vehicle vapor exposure days (grand mean) showed that THC Group 3 also had higher activity on vehicle conditioning days (Fig 4), likely indicating residual THC effects on vehicle exposure days.

\section{Discussion}

In this study, we observed THC vapor-induced CPP in male and female rats at multiple exposure amounts. These data add to prior research examining conditioned effects of THC administered via subcutaneous, intraperitoneal, intravenous injection. While many prior studies using injection have discrepant results, finding either CPP, CPA, or null effects, a recent metaanalysis determined that CPP is most often observed after lower doses (median dose $1 \mathrm{mg} / \mathrm{kg}$ : range $0.075-4 \mathrm{mg} / \mathrm{kg}$ ) whereas higher doses produce CPA (median dose $5 \mathrm{mg} / \mathrm{kg}$; range: 1-20 $\mathrm{mg} / \mathrm{kg}$ ) (Kubilius et al., 2018). Dose-dependent effects of injected THC on anxiety-like behaviors mirror what has been seen with CPP/CPA; low doses produce anxiolytic effects while high 
doses produce anxiogenic effects (Rubino et al., 2007; Valjent et al., 2002). Neurobiologically, low doses of injected THC have been shown to increase extracellular dopamine in rewardrelated brain regions including the nucleus accumbens shell (Tanda et al., 1997) and striatum (Malone and Taylor, 1999); whereas higher doses of THC increase corticosterone via centrally mediated mechanisms (DeVuono et al., 2020; Kokka and Garcia, 1974; Kubena et al., 1971). Therefore, the dose-dependency observed in CPP experiments with injected THC tracks with the dose-dependent effects of THC observed in related behavioral and neurobiological outcomes.

To date, only one prior study has examined conditioned effects of THC vapor, and the study was limited to one sex (male rats). In this study, one THC vapor exposure (10 mg in $16 \mathrm{~L}$ air, delivered over 10-min) prior to conditioning sessions produced a modest preference for the THC-vapor paired side (Manwell et al., 2014). Other exposure conditions tested did not produce CPP or CPA. Specifically, rats exposed to the same dose (10 mg in 16L air), but delivered over a longer period (20-min) did not show a preference for the THC-paired side (Manwell et al., 2014). Additionally, a group receiving the same THC dose over the same duration (10 mg over 10-min), but administered to two rats simultaneously and in a reduced volume (8L air), also did not produce preference, potentially due to less total dose exposure $(10 \mathrm{mg} / 2$ rats vs. $10 \mathrm{mg} / 1$ rat) (Manwell et al., 2014). Similar to our study, no THC vapor exposure amounts tested produced aversion. It is unclear if this similarity reflects a route of administration difference between THC vapor exposure and injected THC, or if thus far, vapor exposure studies have not used a high enough "dose" to produce CPA. We intend on addressing this question in future studies.

There has been one evaluation of sex differences in THC CPP, which used IP injection and observed a place aversion to THC $(0.75 \mathrm{mg} / \mathrm{kg})$ that was no different between the sexes (Hempel et al., 2017). In the present study, we observed that female rats required a greater amount of THC vapor to produce CPP compared with males. This finding was somewhat 
surprising, as the literature suggests that females are more sensitive to cannabinoid effects (Cooper and Craft, 2018), including the reinforcing (Fattore et al., 2007; Fattore et al., 2010) and discriminative effects (Wiley et al., 2017) of synthetic cannabinoid receptor-1 agonists. Indeed, in our previous study, females showed increased appetitive responses after a lower amount of THC vapor exposure compared with males (Moore et al., 2021). However, while females were less sensitive to the conditioned rewarding effects of THC, requiring a higher amount for CPP to develop, this CPP was evident after only 8 conditioning days, while at this stage, CPP in male rats was only near threshold. In addition, females were more sensitive to the locomotor stimulating effects of THC vapor, while males did not show hyperlocomotion. Furthermore, females demonstrated greater resistance to extinction compared with males, who extinguished CPP to THC vapor more rapidly. We did not observe any effects of a drug-primed reinstatement of CPP in male or female rats when re-exposed to the training amount of THC vapor; future studies are needed to explore the use of a a rage of different exposure amounts, as well as cue or stress-primed reinstatement.

\subsection{Conclusions}

In this study, we demonstrated that vaporized THC produced CPP in both male and female rats. There were sex differences in the amount of THC exposure and number of conditioning sessions needed to produce CPP, as well as a sex difference in time to extinction. Further, we saw a striking sex difference in hyperlocomotor response to THC vapor, which was observed in females but not males on THC vapor conditioning days. This is the first demonstration of THC vapor CPP to multiple THC vapor exposure amounts and sex differences in both conditioned effects of THC and extinction in males and female rats. 


\section{Acknowledgements}

This work was supported by the National Institute on Drug Abuse of the National Institutes of Health grant numbers R21DA046154 (EW), the Johns Hopkins University Dalio Fund in Decision Making and the Neuroscience of Motivated Behaviors (EW). The authors also wish to thank Maury Cole and La Jolla Alcohol Research Inc. for development of custom vapor chamber systems and technical assistance. The opinions and assertions expressed herein are those of the author(s) and do not necessarily reflect the official policy or position of the Uniformed Services University or the Department of Defense. 
1. Aguilar, M.A., Rodriguez-Arias, M., Minarro, J., 2009. Neurobiological mechanisms of the reinstatement of drug-conditioned place preference. Brain Res. Rev. 59(2), 253-277.

2. Budney, A.J., Sargent, J.D., Lee, D.C., 2015. Vaping cannabis (marijuana): parallel concerns to e-cigs? Addiction 110(11), 1699-1704.

3. Cooper, Z.D., Craft, R.M., 2018. Sex-Dependent Effects of Cannabis and Cannabinoids: A Translational Perspective. Neuropsychopharmacology 43(1), 34-51.

4. DeVuono, M.V., La Caprara, O., Petrie, G.N., Limebeer, C.L., Rock, E.M., Hill, M.N., Parker, L.A., 2020. Cannabidiol Interferes with Establishment of Delta(9)Tetrahydrocannabinol-Induced Nausea Through a 5-HT1A Mechanism. Cannabis Cannabinoid Res.

5. Fattore, L., Spano, M.S., Altea, S., Angius, F., Fadda, P., Fratta, W., 2007. Cannabinoid self-administration in rats: sex differences and the influence of ovarian function. Br. J. Pharmacol. 152(5), 795-804.

6. Fattore, L., Spano, M.S., Altea, S., Fadda, P., Fratta, W., 2010. Drug- and cueinduced reinstatement of cannabinoid-seeking behaviour in male and female rats: influence of ovarian hormones. Br. J. Pharmacol. 160(3), 724-735.

7. Giroud, C., de Cesare, M., Berthet, A., Varlet, V., Concha-Lozano, N., Favrat, B., 2015. E-Cigarettes: A Review of New Trends in Cannabis Use. Int J Environ Res Public Health 12(8), 9988-10008.

8. Goodman, S., Wadsworth, E., Leos-Toro, C., Hammond, D., International Cannabis Policy Study, t., 2020. Prevalence and forms of cannabis use in legal vs. illegal recreational cannabis markets. Int. J. Drug Policy 76, 102658.

9. Hempel, B.J., Wakeford, A.G., Nelson, K.H., Clasen, M.M., Woloshchuk, C.J., Riley, A.L., 2017. An assessment of sex differences in Delta(9)-tetrahydrocannabinol (THC) taste and place conditioning. Pharmacol. Biochem. Behav. 153, 69-75.

10. Hlozek, T., Uttl, L., Kaderabek, L., Balikova, M., Lhotkova, E., Horsley, R.R., Novakova, P., Sichova, K., Stefkova, K., Tyls, F., Kuchar, M., Palenicek, T., 2017. Pharmacokinetic and behavioural profile of THC, CBD, and THC+CBD combination after pulmonary, oral, and subcutaneous administration in rats and confirmation of conversion in vivo of CBD to THC. Eur Neuropsychopharmacol 27(12), 1223-1237.

11. Kokka, N., Garcia, J.F., 1974. Effects of delta 9-THC on growth hormone and ACTH secretion in rats. Life Sci. 15(2), 329-338.

12. Kubena, R.K., Perhach, J.L., Jr., Barry, H., 3rd, 1971. Corticosterone elevation mediated centrally by delta 1-tetrahydrocannabinol in rats. Eur. J. Pharmacol. 14(1), 89-92.

13. Kubilius, R.A., Kaplick, P.M., Wotjak, C.T., 2018. Highway to hell or magic smoke? The dose-dependence of Delta(9)-THC in place conditioning paradigms. Learn Mem 25(9), 446-454.

14. Lee, D.C., Crosier, B.S., Borodovsky, J.T., Sargent, J.D., Budney, A.J., 2016. Online survey characterizing vaporizer use among cannabis users. Drug Alcohol Depend. 159, 227-233. 
15. Malone, D.T., Taylor, D.A., 1999. Modulation by fluoxetine of striatal dopamine release following Delta9-tetrahydrocannabinol: a microdialysis study in conscious rats. Br. J. Pharmacol. 128(1), 21-26.

16. Manwell, L.A., Charchoglyan, A., Brewer, D., Matthews, B.A., Heipel, H., Mallet, P.E., 2014. A vapourized Delta(9)-tetrahydrocannabinol (Delta(9)-THC) delivery system part I: development and validation of a pulmonary cannabinoid route of exposure for experimental pharmacology studies in rodents. J. Pharmacol. Toxicol. Methods 70(1), 120-127.

17. Moore, C.F., Davis, C.M., Harvey, E.L., Taffe, M.A., Weerts, E.M., 2021. Appetitive, antinociceptive, and hypothermic effects of vaped and injected Delta-9tetrahydrocannabinol (THC) in rats: exposure and dose-effect comparisons by strain and sex. Pharmacol. Biochem. Behav. 202, 173116.

18. Moore, C.F., Stiltner, J.W., Davis, C.M., Weerts, E.M., 2020. Translational models of cannabinoid vapor exposure in laboratory animals. Behav Pharmacol.

19. Morean, M.E., Kong, G., Camenga, D.R., Cavallo, D.A., Krishnan-Sarin, S., 2015. High School Students' Use of Electronic Cigarettes to Vaporize Cannabis. Pediatrics 136(4), 611-616.

20. Murray, J.E., Bevins, R.A., 2010. Cannabinoid conditioned reward and aversion: behavioral and neural processes. ACS Chem Neurosci 1(4), 265-278.

21. Panagis, G., Vlachou, S., Nomikos, G.G., 2008. Behavioral pharmacology of cannabinoids with a focus on preclinical models for studying reinforcing and dependence-producing properties. Curr Drug Abuse Rev 1(3), 350-374.

22. Ramo, D.E., Young-Wolff, K.C., Prochaska, J.J., 2015. Prevalence and correlates of electronic-cigarette use in young adults: findings from three studies over five years. Addict. Behav. 41, 142-147.

23. Rubino, T., Sala, M., Vigano, D., Braida, D., Castiglioni, C., Limonta, V., Guidali, C., Realini, N., Parolaro, D., 2007. Cellular mechanisms underlying the anxiolytic effect of low doses of peripheral Delta9-tetrahydrocannabinol in rats. Neuropsychopharmacology 32(9), 2036-2045.

24. Schauer, G.L., Njai, R., Grant-Lenzy, A.M., 2020. Modes of marijuana use smoking, vaping, eating, and dabbing: Results from the 2016 BRFSS in 12 States. Drug Alcohol Depend. 209, 107900.

25. Shaham, Y., Shalev, U., Lu, L., de Wit, H., Stewart, J., 2003. The reinstatement model of drug relapse: history, methodology and major findings. Psychopharmacology (Berl) 168(1-2), 3-20.

26. Spindle, T.R., Cone, E.J., Goffi, E., Weerts, E.M., Mitchell, J.M., Winecker, R.E., Bigelow, G.E., Flegel, R.R., Vandrey, R., 2020. Pharmacodynamic effects of vaporized and oral cannabidiol (CBD) and vaporized CBD-dominant cannabis in infrequent cannabis users. Drug Alcohol Depend. 211, 107937.

27. Tanda, G., Pontieri, F.E., Di Chiara, G., 1997. Cannabinoid and heroin activation of mesolimbic dopamine transmission by a common mu1 opioid receptor mechanism. Science 276(5321), 2048-2050.

28. Valjent, E., Mitchell, J.M., Besson, M.J., Caboche, J., Maldonado, R., 2002. Behavioural and biochemical evidence for interactions between Delta 9tetrahydrocannabinol and nicotine. Br. J. Pharmacol. 135(2), 564-578. 
29. Varlet, V., Concha-Lozano, N., Berthet, A., Plateel, G., Favrat, B., De Cesare, M., Lauer, E., Augsburger, M., Thomas, A., Giroud, C., 2016. Drug vaping applied to cannabis: Is "Cannavaping" a therapeutic alternative to marijuana? Sci Rep 6, 25599.

30. Wiley, J.L., Lefever, T.W., Marusich, J.A., Craft, R.M., 2017. Comparison of the discriminative stimulus and response rate effects of (Delta9)tetrahydrocannabinol and synthetic cannabinoids in female and male rats. Drug Alcohol Depend. 172, 51-59. 


\begin{tabular}{|c|c|c|c|c|c|c|c|c|}
\hline \multirow[b]{2}{*}{$\begin{array}{l}\text { Vapor } \\
\text { Group }\end{array}$} & \multirow[b]{2}{*}{$\begin{array}{l}\mathbf{N}(50 \% \\
\text { female) }\end{array}$} & \multicolumn{3}{|c|}{ Vehicle Conditioning Days } & \multicolumn{4}{|c|}{ THC Conditioning Days } \\
\hline & & VEH & $\begin{array}{l}\text { Puffs } \\
\text { (\# x } \\
\text { length) }\end{array}$ & $\begin{array}{c}\text { Exposure } \\
\text { Duration } \\
\text { (min) }\end{array}$ & $\begin{array}{c}\text { THC } \\
\text { Concentration } \\
(\mathrm{mg} / \mathrm{ml})\end{array}$ & Puffs & $\begin{array}{c}\text { Exposure } \\
\text { Duration } \\
\text { (min) }\end{array}$ & $\begin{array}{c}\text { Calculated } \\
\text { THC dose } \\
\text { (mg) }\end{array}$ \\
\hline $\mathbf{0}$ & 24 & $100 \% \mathrm{PG}$ & $5 \times 6 s$ & 30 & $0(100 \%$ PG) & $5 \times 6 s$ & 30 & 0 \\
\hline 1 & 24 & $100 \% \mathrm{PG}$ & $5 \times 6 s$ & 30 & 100 & $5 \times 6 s$ & 30 & 7 \\
\hline 2 & 24 & $100 \% \mathrm{PG}$ & $5 \times 6 s$ & 30 & 200 & $5 \times 6 s$ & 30 & 10 \\
\hline 3 & 24 & $100 \% \mathrm{PG}$ & $10 \times 68$ & 30 & 200 & $10 \times 68$ & 30 & 17 \\
\hline
\end{tabular}

Table 1. Conditions of vapor exposure for each group. Each puff was delivered at equally set intervals (e.g. 2.5-5 min apart) across the 30-min session. Total THC drug amounts delievered per rat were calculated based on volume delivered (determined by tank weights before and after sessions) and THC concentration in e-liquid.

\begin{tabular}{|c|c|c|c|c|c|c|c|c|c|c|c|c|c|c|c|c|c|c|c|c|}
\hline Prest & \multicolumn{8}{|c|}{ Place Conditioning Phase 1} & Poost & \multicolumn{8}{|c|}{ Place Conditioning Phase 2} & \multirow{2}{*}{\begin{tabular}{|l|} 
Post \\
nene 2 \\
\end{tabular}} & \multirow{2}{*}{\begin{tabular}{|c|} 
Exfinction \\
none \\
\end{tabular}} & \multirow{2}{*}{$\begin{array}{c}\begin{array}{c}\text { Drug primed } \\
\text { reinstatement }\end{array} \\
\mathrm{THC} \\
\end{array}$} \\
\hline nene & THC & VEH & THC & Ver & THC & ver & THC & ver & none & THC & VEH & THC & VEH & THC & VEH & THC & VEH & & & \\
\hline & 1 & 2 & $\mathbf{3}$ & 4 & 6 & 6 & 7 & $\mathbf{a}$ & & 9 & 10 & 11 & 12 & 13 & 14 & 16 & 16 & & & \\
\hline
\end{tabular}

Figure 1. Conditioned place preference testing timeline. Rats were conditioned across 16 days of vapor exposure with a preference test on day 0, between conditioning days 8-9 and after conditioning day 16. 


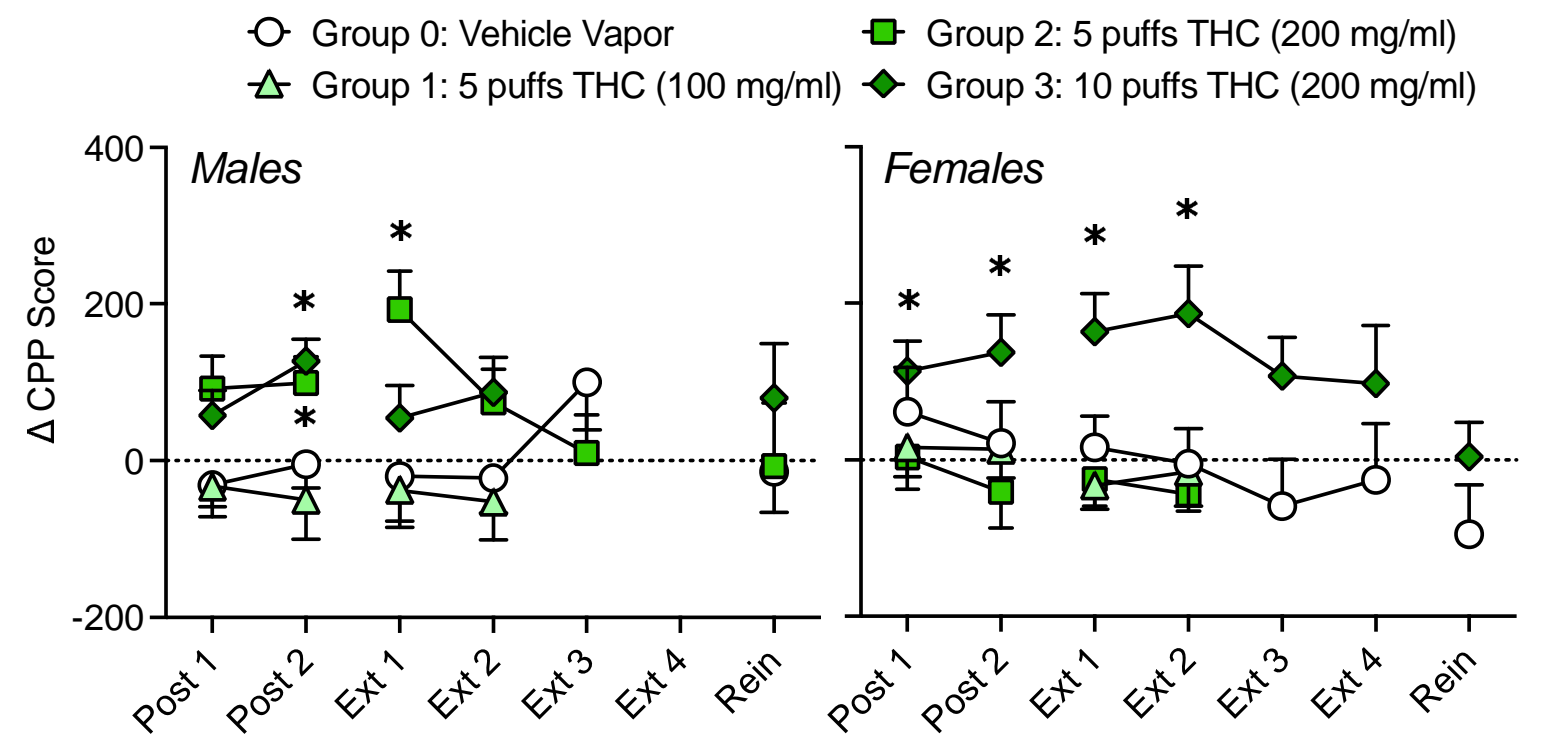

Figure 2. Change in preference score across successive place preference tests. Data shown are group means $\left({ }_{ \pm} \mathrm{SEM}\right)$ for post-tests conducted after each conditioning phases 1 and 2 , followed by extinction and reinstatement tests. * represents a change in CPP score (CPP Score Pre-Test - CPP Score Post-Test $)>0(p>0.05)$, determined by a one-sample t-test. 


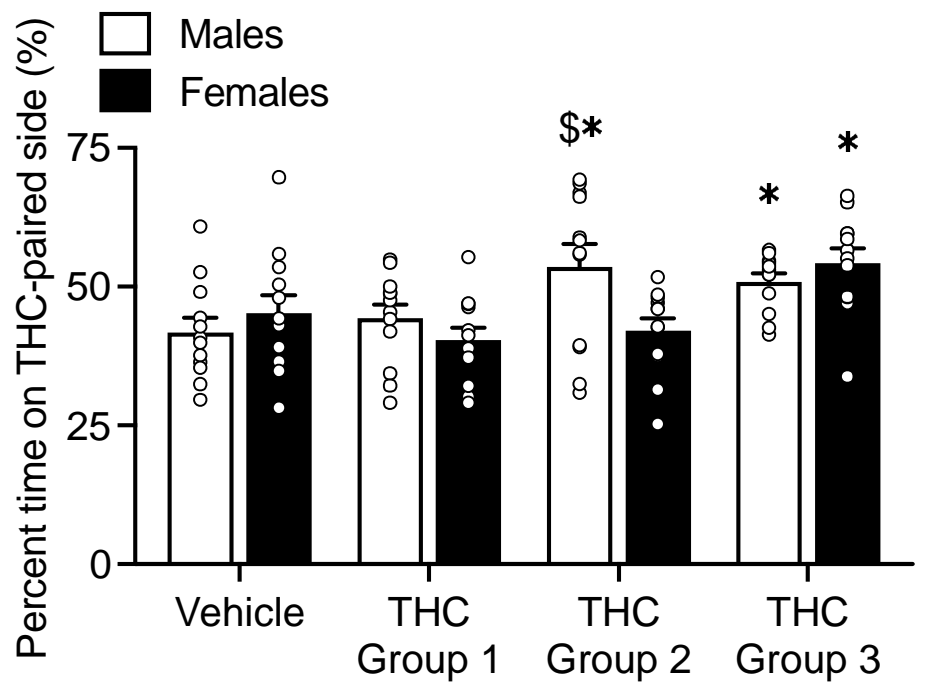

Figure 3. Percent of time spent on the drug-paired side. Asterisks $\left(^{*}\right)$ indicate a difference from the vehicle group of the same sex (p’s $\leq 0.05)$; dollar sign $(\$)$ indicates a sex difference within that vapor group $(p=0.02)$. 
- Group 0: Vehicle Vapor $\quad \square$ Group 2: 5 puffs THC $(200 \mathrm{mg} / \mathrm{ml})$
$\triangle$ Group 1: 5 puffs THC (100 mg/ml) $\diamond$ Group 3: 10 puffs THC $(200 \mathrm{mg} / \mathrm{ml})$

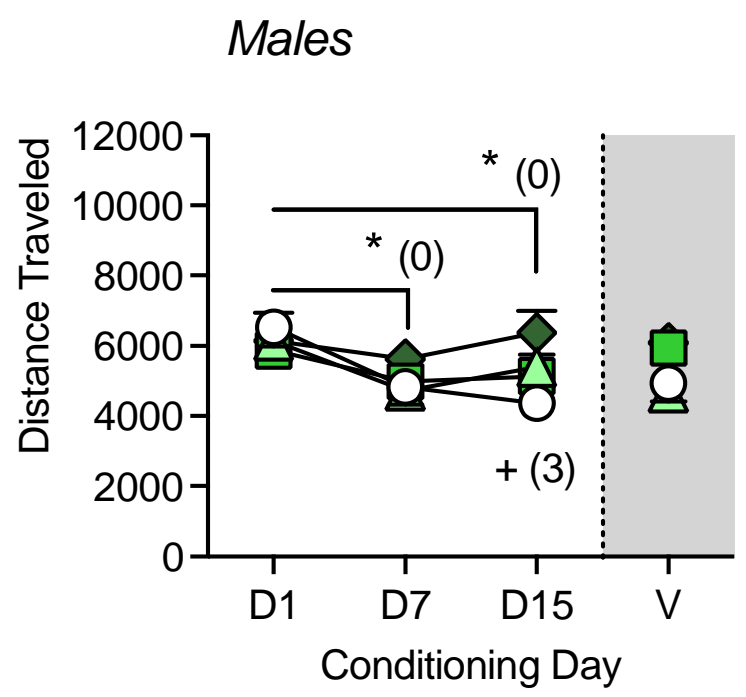

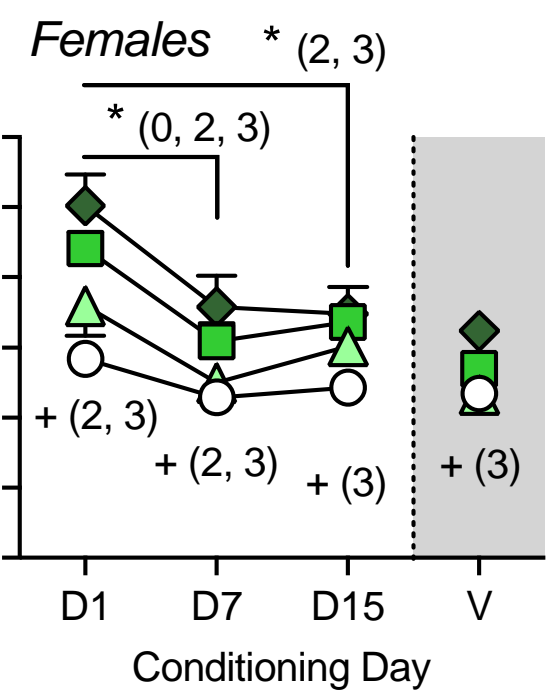

Figure 4. Distance traveled on conditioning days 1, 7, and 15 (white background) compared with the mean distance traveled on vehicle conditioning days (gray background). Asterisks $\left(^{*}\right)$ denote differences from Day 1 (D1) for groups 0-3 (p's<0.05). Pound signs (\#) denote THC group differences (1-3) from the Vehicle vapor control group (p's<0.05). 